\title{
OPEN Template activating factor-I epigenetically regulates the TERT transcription in human cancer cells
}

\author{
Kohsuke Kato ${ }^{1 \bowtie}$, Atsushi Kawaguchi ${ }^{1,2,3}$ \& Kyosuke Nagata ${ }^{4 凶}$ \\ Telomere, the terminus of linear chromosome in eukaryotes, is composed of specific repeat DNA \\ which is mainly synthesized by a protein complex called telomerase. The maintenance of telomere \\ DNA is important for unlimited proliferative capacity of cancer cells. The telomerase activity is \\ controlled by the expression level of telomerase reverse transcriptase (TERT), a catalytic unit of \\ telomerase, in some species including human. Therefore, to reveal the regulatory mechanisms of the \\ transcription of TERT gene is important for understanding the tumor development. We found that \\ template activating factor-I (TAF-I), a multifunctional nuclear protein, is involved in the transcriptional \\ activation of TERT for the maintenance of telomere DNA in HeLa cells. TAF-I maintains the histone \\ H3 modifications involved in transcriptional activation and hypomethylated cytosines in CpG \\ dinucleotides around the transcription start site (TSS) in the TERT gene locus. Collectively, TAF-I is \\ involved in the maintenance of telomere DNA through the regulation of TERT transcription, then \\ consequently the occurrence and/or recurrence of cancer cells.
}

Telomeres are located at the ends of chromosome and have specific repetitive DNA sequence in eukaryotes. Because DNA polymerase cannot synthesize 5'-terminal regions, telomere DNA is gradually shortened every DNA replication in most terminally differentiated human cells (end replication problem) ${ }^{1}$. The shortened telomere DNAs are recognized by cellular DNA damage sensor proteins, then the DNA damage-signaling pathways induce the irreversible cell cycle-arrest through the activation of several cell cycle regulators such as p53. Thus, after repeating cell divisions, terminally differentiated cells usually enter the cell cycle-arrested state, called senescence, due to the limited dividing capacity ${ }^{2}$. This suggests that unlimited telomere DNA synthesis is essential to acquire immortality which consequently takes a chance to proceed the cancer.

The telomere DNA is synthesized by telomerase, a ribonucleoprotein complex consisting of a catalytic subunit telomerase reverse transcriptase (TERT), a template RNA telomerase RNA component (TERC), and several other proteins ${ }^{3}$. Telomerase is usually activated in stem cells and cancer cells. The expression level of TERT is highly regulated at both transcriptional and post-transcriptional level. The transcription from the TERT promoter is a primary determinant of the telomerase activity depending on cell types and cell states. As summarized in a review by Gaspar et al., telomerase reactivation is observed in approximately $90 \%$ of human cancer cells through the upregulation of TERT transcription ${ }^{4}$. A variety of transcription factors regulate the TERT transcription. Sp1 and c-Myc function as major transcriptional activators of TERT through their bindings on the TERT promoter region $^{5}$. In contrast, several transcription factors such as CTCF and WT1 negatively regulate the transcription of $T E R T^{6,7}$. Several nucleotide mutations are frequently introduced in the TERT promoter region and implicated in telomerase reactivation in cancer cells by the de novo binding sites for ETS family transcription factors such as $\mathrm{GABP}^{8-10}$. In addition to such somatic mutations, amplification of TERT gene $\mathrm{e}^{11,12}$ and rearrangement of TERT locus ${ }^{13,14}$ directing its transcriptional activation are also reported during cancer development. In addition to genetic mechanism, the transcription of TERT is also regulated by epigenetic mechanisms. TERT gene contains $\mathrm{CpG}$ islands located at the region from $838 \mathrm{bp}$ upstream of the first AUG codon to the end of exon $2^{15}$. It is well known that 5-methyl-cytosine $(5 \mathrm{mC})$ in $\mathrm{CpG}$ dinucleotides functions as a pivotal epigenetic mark in gene silencing. In general, the hypomethylated status around the TSS in the TERT promoter is required for its transcriptional activation ${ }^{16}$. In contrast, as summarized in a review by Lee et al., many previous studies reveal that the hypermethylation in TERT locus is correlated with high expression level of telomerase in a variety of cancer cells ${ }^{17}$. In particular, a $433 \mathrm{bp}$-long genomic region including $52 \mathrm{CpG}$ sites located upstream of the core

\footnotetext{
${ }^{1}$ Department of Infection Biology, Faculty of Medicine, University of Tsukuba, 1-1-1 Tennodai, Tsukuba 305-8575, Japan. ${ }^{2}$ Transborder Medical Research Center, University of Tsukuba, Tsukuba, Japan. ${ }^{3}$ Microbiology Research Center for Sustainability, University of Tsukuba, Tsukuba, Japan. ${ }^{4}$ Faculty of Medicine, University of Tsukuba, Tsukuba, Japan. ${ }^{\varpi}$ email: kkato@md.tsukuba.ac.jp; knagata@md.tsukuba.ac.jp
} 
promoter in TERT locus, called the TERT hypermethylated oncological region (THOR), is highly methylated and involved in the cancer-associated transcription of the TERT ${ }^{18}$. The hypermethylation of THOR promotes the TERT transcription by inhibiting the binding of transcription repressors such as CTCF and WT $1{ }^{17}$. Not only DNA methylation, but also several histone modifications involved in transcriptional regulation are also important epigenetic marks to regulate the TERT transcription. Acetylation of histone H3 K9 and K14 (K9K14ac) ${ }^{19,20}$ and tri-methylation of histone $\mathrm{H} 3 \mathrm{~K} 4(\mathrm{~K} 4 \mathrm{me} 3)^{19,21}$ promotes the transcriptional activation of TERT through the formation of open chromatin structure. Upon transcriptional repression, tri-methylations of histone $\mathrm{H} 3 \mathrm{~K} 9$ $(\mathrm{K} 9 \mathrm{me} 3)^{20}$ or K27 (K27me3) $)^{15,21}$ are introduced in the TERT gene locus to form closed chromatin structures. However, the detailed mechanism how the TERT gene activity is epigenetically regulated remains unclear.

Template activating factor-I (TAF-I) was originally identified as a host factor that activates adenovirus DNA replication and transcription through the remodeling of chromatin-like viral genome DNA-protein complexes ${ }^{22}$. Two subtypes of TAF-I, TAF-I $\alpha$ and TAF-I $\beta$, are expressed from the TAF-I gene locus using two alternative promoters $^{23}$. TAF-I has a histone chaperone activity against histone $\mathrm{H} 3$ and $\mathrm{H} 1$ in vitro ${ }^{24-27}$. In particular, we found that TAF-I is associated with several histone H1 variants and regulates its chromatin-binding dynamics in the nucleus ${ }^{26}$. TAF-I is involved in transcriptional regulation of interferon-stimulated genes (ISGs) through its histone $\mathrm{H} 1$ chaperone activit ${ }^{28}$. TAF-I also regulates epigenetic marks related to transcription without the histone chaperone activity. TAF-I inhibits the acetylation of histone $\mathrm{H} 3$ as a subunit of inhibitor of histone acetyltransferase (INHAT) complex ${ }^{29,30}$. Furthermore, it is reported that TAF-I indirectly regulates the level of DNA methylation through upregulating the expression of ten-eleven translocation 1 (TET1), a hydroxylation enzyme of methylated cytosine, for the DNA demethylation ${ }^{31}$. However, how each gene activity individually controlled by TAF-I through epigenetic mechanisms is still unclear.

Here, we found that TAF-I maintains the telomere integrity through the epigenetic regulation of the TERT gene transcription in human cancer cells. The transcription of TERT was downregulated by knock down (KD) of TAF-I. Histone H3 K9K14ac, K4me3, and DNA methylation around the TSS in TERT promoter are regulated by TAF-I. Collectively, we proposed that TAF-I is involved in the occurrence and/or recurrence of cancer cells by telomerase reactivation through the epigenetic mechanisms.

\section{Results}

Expression level of TERT mRNA, telomerase activity, and telomere DNA length are impaired in TAF-I KD HeLa cells. From previous our microarray analyses, we have identified TERT as one of downregulated genes at mRNA level in shRNA-mediated TAF-I KD HeLa cell lines ${ }^{32}$. To confirm this, we examined the amount of TERT mRNA in TAF-I KD HeLa cell lines ${ }^{33}$ by quantitative RT-PCR (Q-RT-PCR) analyses. We used two HeLa cell lines (clone \#7 and \#8) expressing both TAF-I $\alpha$ and TAF-I $\beta$ at the similar levels of intact HeLa cells, defined as WT cells, and two other cell lines (clone \#4 and \#13), in which TAF-I expression is strongly reduced compared to WT cells, defined as TAF-I KD cells (Fig. 1A, Supplementary Fig. 1A-E). The amount of the TERT mRNA in both clone \#4 and \#13 TAF-I KD cells was reduced to approximately $20 \%$ of WT cells (Fig. 1B). We also examined the amount of TERC RNA in both WT and TAF-I KD cells (Fig. 1C). Although we found that the amount of TERC RNA in clone \#4 cells was slightly reduced compared to WT cells, the reduction of TERT mRNA was higher than that of TERC RNA. Next, we examined the enzymatic activity of telomerase in TAF-I KD cells by telomeric repeat amplification protocol (TRAP) assays. We performed TRAP assays with appropriate PCR cycles to prevent a saturation of PCR amplicons (Supplementary Figs. 2A and 8A). The efficiently amplified PCR products was detected in the cell lysates prepared from WT cells (Fig. 1D, lanes 5-8, and Supplementary Fig. 8B), and the amplified products were not observed in the RNase A-treated or heat-denatured cell lysates (Fig. 1D, lanes 11 and 12), indicating that the amplification of PCR products is dependent on the telomerase activity. In contrast, the amount of PCR products decreased in the cell lysates prepared from TAF-I KD cells compared with that of WT cells (Fig. 1D, lanes 3-4 and lanes 9-10), suggesting that the telomerase activity in TAF-I KD cells are impaired possibly due to the reduction of the TERT mRNA. These results suggest that TAF-I is involved in the maintenance of the telomerase activity in HeLa cells through upregulating TERT expression. Notably, the amount of $\beta$-actin in the cell lysates was not changed between each cell line, suggesting that the cell lysates were prepared from each cell line to similar extents (Supplementary Figs. 1F,G, 2B). For more quantitative analyses of telomerase activity, we also performed Q-PCR-based TRAP (qTRAP) assay. The telomerase activity in TAF-I KD cells is also lower than that in WT cells similarly observed in Fig. 1D (Fig. 1E). Furthermore, we examined the length of telomere repeat DNA in TAF-I KD cells by telomere-FISH assays ${ }^{34}$. The relative intensity of telomere repeat DNA in TAF-I KD cells was lower than that of WT cells (Fig. 1F, Supplementary Figs. 3, and 4A-D). We also performed a TRF assay to evaluate the length of telomere repeat DNA. In both WT cells, we detected approximately $14 \mathrm{kbp}$-peaked TRFs (Supplementary Figs. 5A,B, 8C,D). In TAF-I $\mathrm{KD} \# 4$ cells, approximately $4.5 \mathrm{kbp}$-peaked TRF was clearly detected (Supplementary Fig. 5A,B). In contrast to \#4 cells, we detected relatively longer TRF DNA in TAF-I KD \#13 cells, but it was actually shortened compared to that in WT cells (Supplementary Fig. 5A,B). These results suggest that telomere repeat DNA in TAF-I KD HeLa cells becomes relatively shorter than that in WT cells. Furthermore, we examined whether TAF-I KD affects cell growth of HeLa cell lines. We found that the cell growth of TAF-I KD cells is moderately retarded compared to that in control cells after $72 \mathrm{~h}$ post cell plating (Fig. 1G). It is possible that TAF-I KD affects cell growth through the regulation of TERT transcription in HeLa cells.

The reduction of TERT expression in TAF-I KD cells is not due to the instability of TERT mRNA. The amount of TERT mRNA is regulated at both transcriptional and post-transcriptional levels. It is reported that the stability of TERT mRNA is upregulated by poly $(\mathrm{C})$ binding proteins through its binding to the $3^{\prime}$-UTR of TERT mRNA ${ }^{35}$. To clarify whether the reduction of TERT mRNA in TAF-I KD cells is caused by post- 


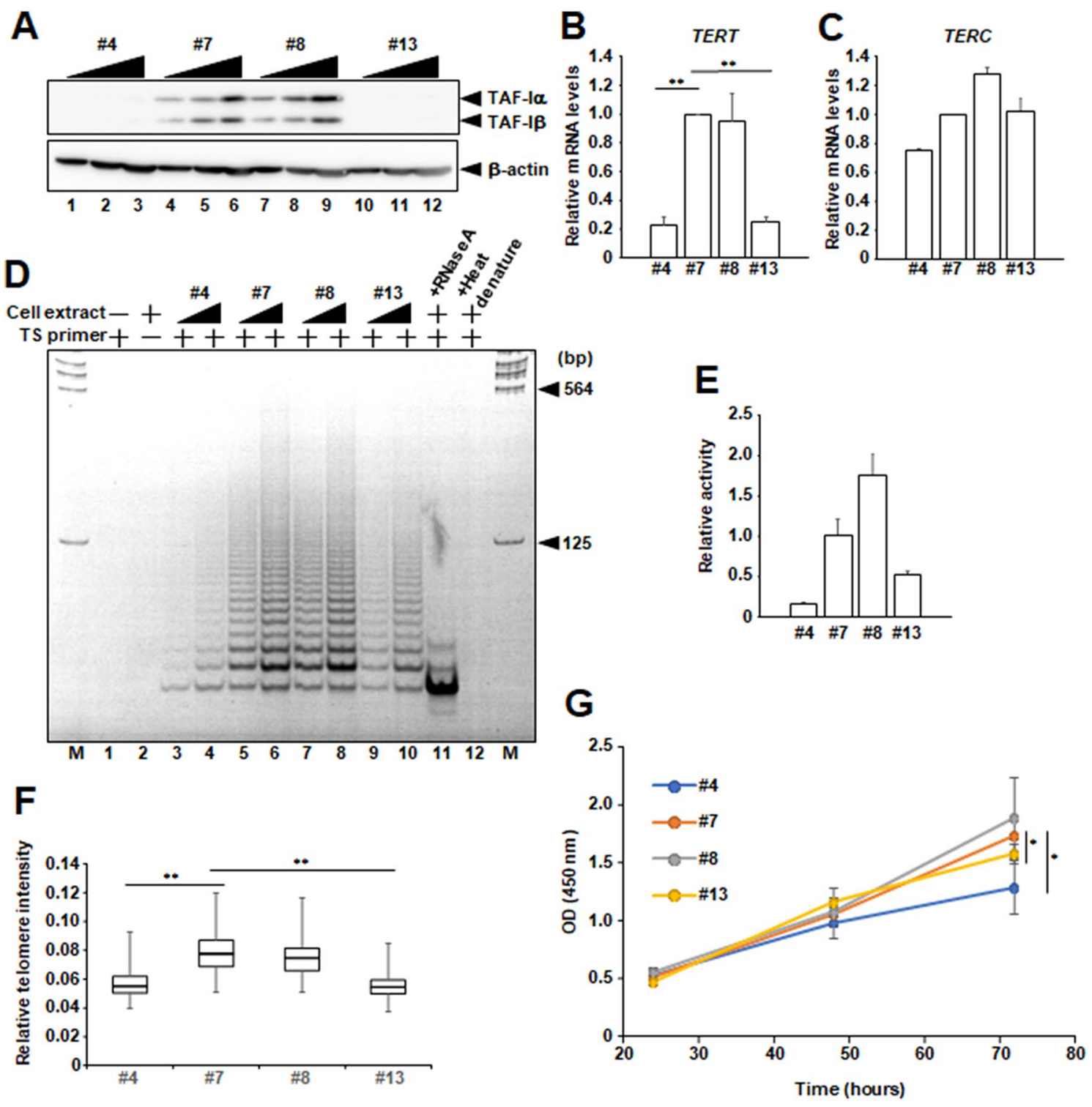

Figure 1. The TERT expression and telomerase activity is downregulated in TAF-I KD HeLa cell lines. (A) TAF-I $\alpha$ and TAF-I $\beta$ expression levels were examined by western blotting analyses. Total cell extracts prepared from WT (\#7: lanes 4-6, and \#8: lanes 7-9) and TAF-I KD (\#4: lanes 1-3, and \#13: lanes 10-12) HeLa cell lines were separated on $10 \%$ SDS-PAGE followed by western blotting analyses using anti-TAF-I $\alpha / \beta$ and anti$\beta$-actin antibodies. Five, ten, and twenty $\mu$ g of total protein prepared from each cell line was loaded. (B,C) The expression level of TERT mRNA and TERC RNA was examined by Q-RT-PCR analyses in each HeLa cell lines. The $18 \mathrm{~S} r R N A$ was used for normalization as an internal control. Values represent the mean \pm SD $(\mathrm{n}=3) .{ }^{*} P<0.01$ relative to \#7. (D) Telomerase activity was examined by TRAP assay. Each cell extract derived from $5.0 \times 10^{2}$ cells (lanes $3,5,7$, and 9) and $2.5 \times 10^{3}$ cells (lanes $4,6,8$, and 10 ) was incubated as described in "Materials and methods". Reactions were done without cell extract (lane 1) or TS primer (lane 2) as negative controls. Cell extract pre-treated with RNase A (lane 11) or heat-denatured (lane 12) was used to confirm the telomerase-dependency on reactions. (E) Q-TRAP assay. Each cell extract derived from $1.0 \times 10^{2}$ cells was incubated in reaction mix and subjected to Q-PCR analyses as described in "Materials and methods". Values represent the mean $\pm S D(n=3)$. (F) Relative telomere intensity of WT and TAF-I KD cells. A value of total telomere intensity in one cell nucleus was normalized by dividing it with a value of total DAPI signal in same cell nucleus, then plotted as a box plot. At least, images of more than 130 cells were taken and analyzed in each cell clone. ${ }^{* \star} P<0.01$ relative to \#7. (G) Cell growth assay in HeLa cell lines. The horizontal line indicates time after plating cells, and the vertical line indicates the absorbance at $450 \mathrm{~nm}$ after adding WST- 8 reagents. Values represent the mean $\pm \mathrm{SD}(\mathrm{n}=3) .{ }^{*} P<0.05$ relative to $\# 7$. 
A

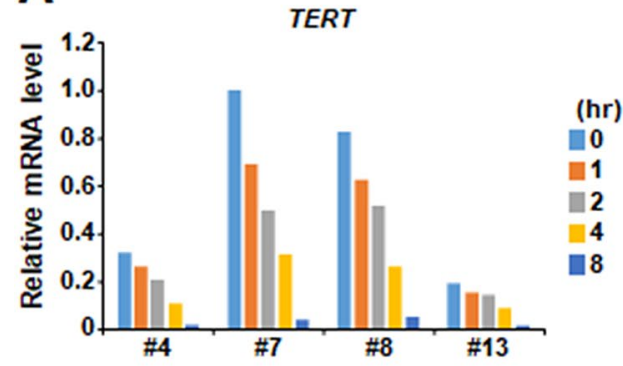

C

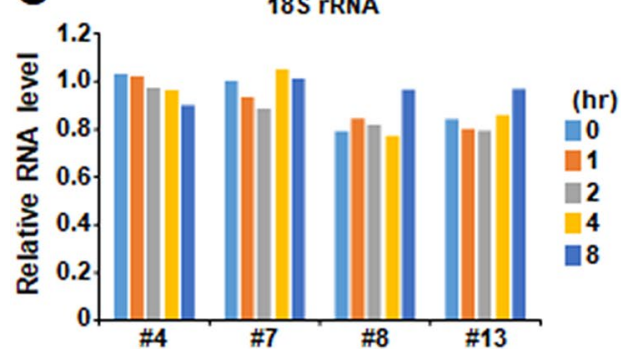

B

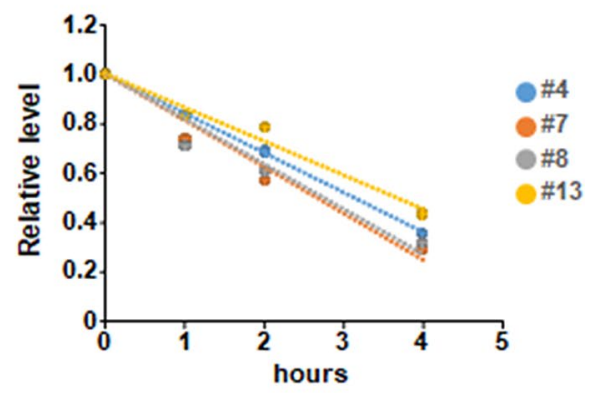

Figure 2. Stability of the TERT mRNA was not so different between WT and TAF-I KD cells. (A,C) The expression level of TERT mRNA in WT and TAF-I KD cells which were treated with actinomycin D was examined by Q-RT-PCR analyses. WT and TAF-I KD cells treated with actinomycin D for 1, 2, 4 and $8 \mathrm{~h}$ were collected and subjected to total RNA purification. Each cell just before treating with actinomycin D was also collected as a $0 \mathrm{~h}$ sample. Q-RT-PCR analyses were performed with each purified total RNA to examine the expression level of TERT mRNA and 18S rRNA. Relative expression level of TERT mRNA and 18S rRNA was plotted as indicated graphs $(\mathbf{A}, \mathbf{C})$, respectively. (B) The value of TERT mRNA was normalized by dividing it with that of $18 \mathrm{~S}$ rRNA. Each relative value when the normalized value of $0 \mathrm{~h}$ sample was set to be 1.0 was represented.

was examined by Q-RT-PCR. The amount of TERT mRNA in each cell line gradually decreased to a similar extent in a time-dependent manner by adding actinomycin D (Fig. 2A,B), while the amount of 18S rRNA was unchanged (Fig. 2C). This suggests that the stability of TERT mRNA is not impaired by TAF-I KD.

Transcription from transiently transfected TERT promoter-driven reporter gene is not downregulated in TAF-I KD cells. TAF-I is involved in the transcriptional regulation of a variety of genes through multiple mechanisms. TAF-I directly regulates the DNA binding activity of several transcription factors such as Sp1 and regulates the transcription of their downstream genes ${ }^{36,37}$. In addition, it was reported that TAF-I $\beta$ promotes the stability of $\mathrm{c}-\mathrm{Myc}$ through the inhibition of PP2A-mediated dephosphorylation ${ }^{38}$. Therefore, there is a possibility that TAF-I directly regulates the transcription of TERT through Sp1 and/or c-Myc. We performed reporter gene assays using plasmids harboring human TERT promoter-driven firefly luciferase (Luc) gene (pTERT-Luc). pTERT-Luc plasmids having different length of human TERT promoter ${ }^{39}$ were examined (Fig. 3A). Both Sp1 and c-Myc are critical activators of the TERT promoter on these constructs ${ }^{39}$. The luciferase activity in TAF-I KD clone \#13 cells transfected with pTERT-Luc-1375, -776, and -181 was comparable to that of WT cells, respectively (Fig. 3B). In addition, the luciferase activity in TAF-I KD clone \#4 cells transfected with pTERT-Luc-1375, and -776 was not reduced, rather showed a little higher activity than that of WT clone \#7 cells, respectively (Fig. 3B). Note that a plasmid lacking the transcription start site (pTERT-Luc +19$)$ did not show any luciferase activity, indicating that the TERT promoter on reporter plasmids is properly activated in this assay condition (Fig. 3B). Thus, it is possible that the reduction of the TERT promoter activity by TAF-I KD is not reconstructed on transiently transfected reporter plasmids in HeLa cells. It is suggested that transiently transfected plasmid DNA does not form a well-organized chromatin structures because of irregular histone binding ${ }^{40}$. Considering these facts, we assumed that TAF-I is involved in the transcriptional activation of TERT on chromatinized DNA through the epigenetic mechanisms.

The methylation level of CPG dinucleotides around TSS of the TERT is increased by TAF-I KD. The cytosine methylation in $\mathrm{CpG}$ dinucleotides generally represses transcription by recruiting transcriptional repressors or co-repressors onto promoter regions including the TERT gene. However, DNA methylations in THOR and gene body region in the TERT gene locus are involved in its transcriptional activation by suppressing the binding of several transcription repressors including CTCF. Thus, it is suggesting that the CpG methylation-mediated gene regulation has more complicated molecular functions in the regulation of TERT transcription and the cancer progression ${ }^{16,18}$. We next tested the cytosine methylation of CpG islands (CGIs) located at the TERT promoter in TAF-I KD cells by bisulfite sequencing analyses. The TERT promoter region contains two CGIs between nucleotide positions - 130 to + 143 (CGI-1), and between nucleotide positions - 389 to - 155 

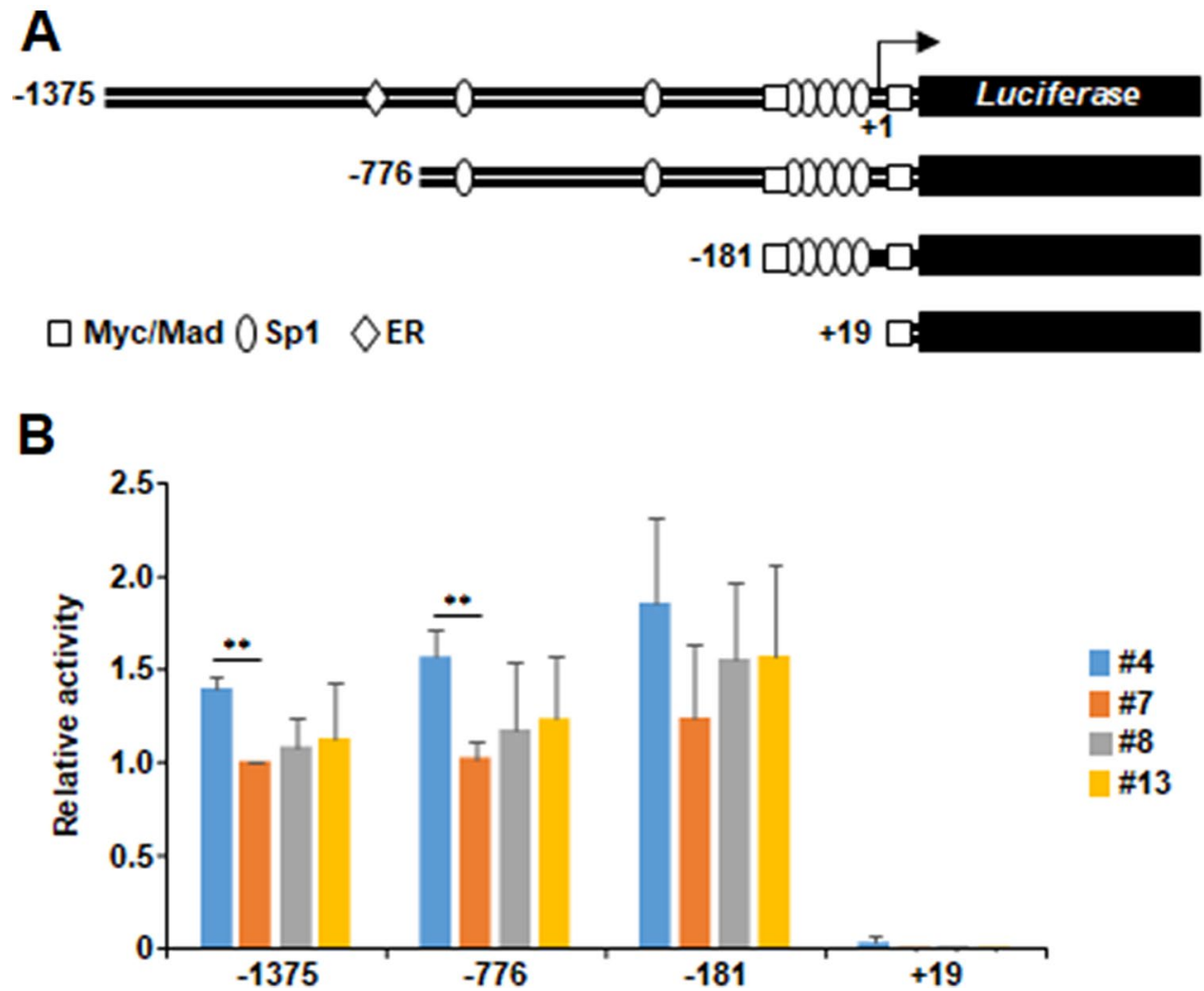

Figure 3. Transcription from transiently transfected pTERT-Luc reporter plasmids could not reconstitute the downregulation of endogenous TERT transcription by TAF-I KD. (A) Schematic diagrams of pTERT-Luc reporter genes. The upstream and downstream nucleotide positions from transcription start site $(+1)$ of each pTERT-Luc plasmid are shown. Binding sites for major transcription factors such as Myc/Mad, Sp1, and ER are represented. (B) Results of luciferase assay. Each pTERT-Luc reporter plasmid together with pEF1a-RLuc control plasmid were transfected into WT and TAF-I KD cells. Renilla luciferase was used as an internal control. After $48 \mathrm{~h}$ post transfection, cells were collected, and cell extract was prepared and subjected to measure both firefly and renilla luciferase activities. Each value of firefly luciferase activity was normalized by dividing it with that of renilla luciferase. Each relative value when the normalized value of pTERT-1375-Luc-transfected clone \#7 cells was set to be 1.0 was represented. Values represent the mean $\pm \mathrm{SD}(\mathrm{n}=3) .{ }^{\star *} P<0.01$ relative to \#7.

(CGI-2) relative to the TSS (Fig. 4A) ${ }^{20}$. Note that CGI-2 is completely included in THOR ${ }^{18}$. In WT cells, the level of CpG methylations at nucleotide positions - 100 to - 50 within core promoter region of CGI-1 was lower than that of other regions including CGI-2 (Fig. 4B). It suggests that the low level of CpG methylation around the TSS is important for transcriptional activation of TERT. We found that the CpG methylation level around TSS in CGI-1 increased about 1.5- to 3-fold by TAF-I KD, although CGI-2 does not show significant differences between WT and TAF-I KD cells (Fig. 4B,C). These results suggest that TAF-I activates the TERT expression by decreasing the level of $\mathrm{CPG}$ methylation around the TSS, but not upstream THOR in the TERT locus. Previously, it was reported that over-expression of TAF-I $\beta$ indirectly promotes the global CpG demethylation of genomic DNA through the upregulation of TET1 expression in HEK293 cells ${ }^{31}$. However, the TET1 was poorly expressed in HeLa cells in contrast to other cancer cells (Supplementary Fig. 6A,B). Therefore, we also examined the level of 5-hydroxymethyl-cytosine (5hmC) at the CGI-1 in TERT by methylated DNA-immunoprecipitation (MeDIP) assays. As a result, we could not observe the reduction of $5 \mathrm{hmC}$ in TAF-I KD cells compared to that in WT cells (Supplementary Fig. 7), suggesting that TAF-I-mediated maintenance of low level CpG methylation at CGI-1 in TERT is independent of the upregulation of TET1 expression.

To address whether the fluctuation of CpG methylation at CGI-1 is correlated with transcriptional activity of TERT, we performed Q-RT-PCR and bisulfite sequencing analyses in TAF-I KD cells treated with decitabine (5-aza-2'-deoxycytidine), a DNA methyltransferase inhibitor. The amount of TERT mRNA in decitabine-treated TAF-I KD cells was approximately fourfold increased compared to that in DMSO-treated cells (Fig. 5A). In parallel, the level of $\mathrm{CpG}$ methylation in decitabine treated-TAF-I KD \#4 cells was broadly decreased to approximately one-half compared to that in DMSO-treated (Fig. 5B), suggesting that CpG methylation at CGI-1 of TERT regulates its transcriptional activity. Taken together, these results suggests that TAF-I is involved in transcriptional activation of TERT through the maintenance of lower CpG methylation around TERT TSS.

The histone modifications involved in transcriptional activation were down-regulated on the TERT promoter by TAF-I KD. In general, CpG methylation and histone modifications cooperatively regu- 

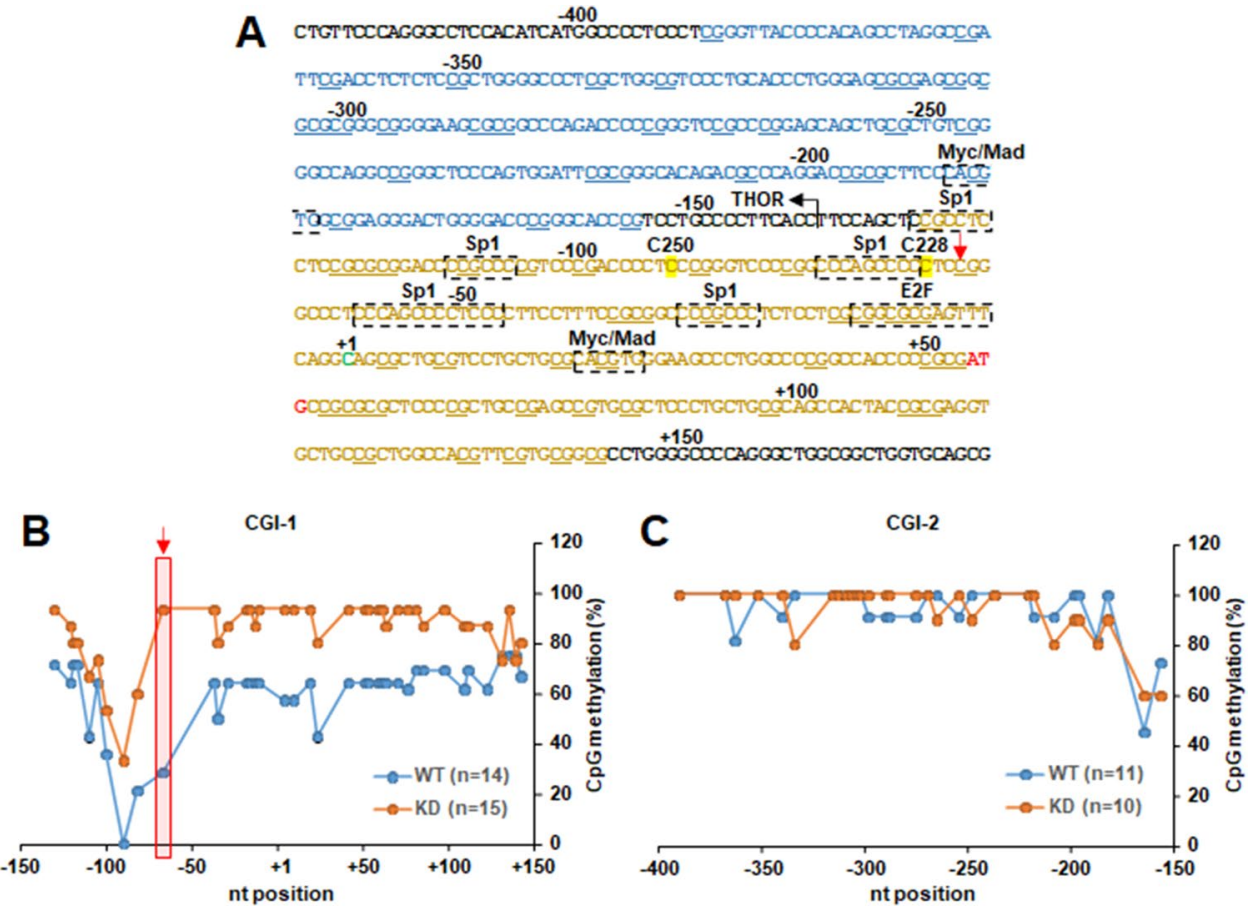

Figure 4. The level of CpG dinucleotides methylation around the TERT TSS was higher in TAF-I KD cells compared to that of WT cells. (A) The DNA structure in human TERT locus analyzed in bisulfite sequencing analyses. DNA sequence corresponding to the region from -424 to +176 nucleotide positions of TSS in human TERT locus is represented. CpG island region-1 (CGI-1) and CGI-2 are shown by yellow and blue letters, respectively. All analyzed $\mathrm{CpG}$ dinucleotides are indicated by under bars. The nucleotide positions of transcription site $(+1)$ and a translation initiation codon (first ATG) of the TERT are shown by green and red letters, respectively. The nucleotide positions of C228T and C250T mutations are highlighted with yellow marker. Binding sites for major transcription factors such as Myc/Mad, Sp1, and E2F are indicated by dashed lined boxes. The start site of THOR is indicated by a bended arrow. The position of cytosine showing the most marked difference of methylation level between WT and TAF-I KD cells is indicated by a red arrow. (B,C) Bisulfite sequencing analyses. The methylation level of each CpG dinucleotide in CGI-1 (B) and CGI-2 (C) of the TERT locus was compared between WT and TAF-I KD cells. The horizontal line indicates nucleotide positions from the TERT TSS, and the vertical line indicates the percentage of CpG methylation. In (B), the position of cytosine showing the most marked difference of methylation level between WT and TAF-I KD cells is indicated by a red arrow and a shadowed box (please note a red arrow in (A), too). Total numbers of DNA clone used for sequencing were 14 (clone \#7: 8, and \#8: 6) for WT and 15 (clone \#4: 9, and \#13: 6) for TAF-I KD in CGI-1 (B), and 11 (clone \#7: 7, and \#8: 4) for WT and 10 (clone \#4: 6, and \#13: 4) for TAF-I KD in CGI-2 (C), respectively.

lates the transcription through the several mechanistic interactions ${ }^{41}$. It is reported that histone H3 K9K14ac and K4me3 are involved in transcriptional activation of TERT, while histone H3 K9me3 and K27me3 oppositely repress it. We next examined the transcription-related histone modifications on the TERT promoter in TAF-I KD cells by chromatin immunoprecipitation (ChIP) assays. We measured the amount of histone H3 K9K14ac and $\mathrm{K} 4 \mathrm{me} 3$ as markers for actively transcribed gene, and histone $\mathrm{H} 3 \mathrm{~K} 27 \mathrm{me} 3$ as a marker for transcriptionally silenced gene. Although the amount of histone H3 around the TERT TSS in TAF-I KD clone \#4 cells was slightly increased, that in TAF-I KD clone \#13 cells was not changed (Fig. 6A), suggesting that the amount of histone $\mathrm{H} 3$ is not involved in the downregulation of the TERT transcription in TAF-I KD cell lines. The level of histone H3 K9K14ac and K4me3 in both TAF-I KD cells was reduced to about 30 to $40 \%$ of that in WT cells (Fig. 6A,B). In contrast, histone $\mathrm{H} 3 \mathrm{~K} 27 \mathrm{me} 3$, a marker of transcriptionally silenced gene, was not dramatically changed by TAF-I KD (Fig. 6A,B). These results strongly suggest that TAF-I is involved in the transcriptional activation of TERT through the upregulation of histone modifications implicated in the transcriptional activation such as histone H3 K9K14ac and K4me3. In addition, we performed ChIP assay to examine the amount of RNA polymerase II (pol II), Sp1 and c-Myc to reveal the effect of TAF-I KD on transcription machineries. The amount of pol II in TAF-I KD cells was reduced to about $50 \%$ of that in WT cells (Fig. 6C). The amount of promoter-bound Sp1 but not c-Myc in TAF-I KD cells was also reduced to about 50 to $60 \%$ of that in WT cells (Fig. 6D). These results suggest that the reduction of chromatin-bound Sp1 through the changes of epigenetic marks is one of causes on TAF-I KD-dependent downregulation of TERT transcription.

Histone $\mathrm{H} 1$ is generally involved in transcriptional repression through the formation of highly packed chromatin structure ${ }^{42}$. We previously revealed that TAF-I is involved in the transcriptional repression through the deposition of histone H1.2 onto the promoter region of ISGs ${ }^{28}$. It is also reported that histone H1 suppresses the 
A

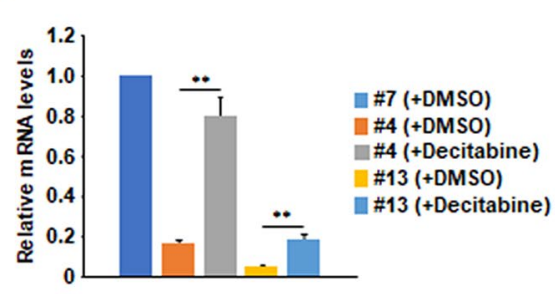

B

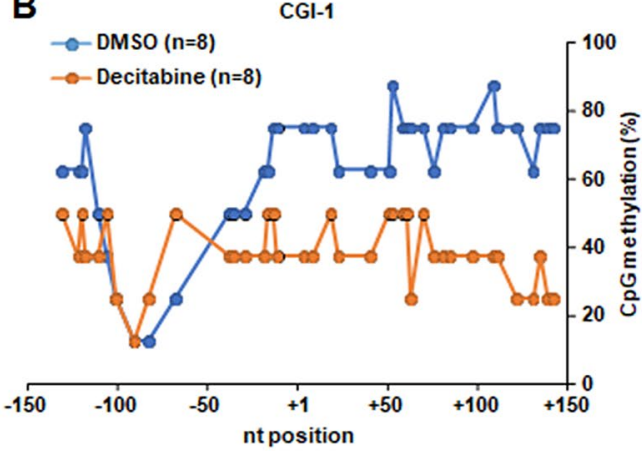

Figure 5. Decitabine-treatment upregulated TERT transcription through the reduction of CpG methylation at CGI-1 of TERT in TAF-I KD cells. (A) The expression level of TERT mRNA was examined in decitabine-treated HeLa cell lines. WT clone \#7, TAF-I KD clone \#4 and clone \#13 cells were cultured in medium containing $0.003 \%$ DMSO (vehicle) or $30 \mu \mathrm{M}$ decitabine for 4 days. Total RNA was isolated from each cell, and was subjected to Q-RT-PCR analyses to examine the expression level of TERT mRNA. The $18 \mathrm{~S}$ rRNA was used for normalization as an internal control. Values represent the mean $\pm \mathrm{SD}(\mathrm{n}=3) .{ }^{* *} P<0.01$ relative to each TAF-I KD cells treated with DMSO. (B) Bisulfite sequencing analyses. The methylation level of each CpG dinucleotide at CGI-1 of TERT was compared between DMSO or decitabine-treated TAF-I KD clone \#4 cells. The horizontal line indicates nucleotide positions from the TERT TSS, and the vertical line indicates the percentage of CpG methylation.

transcription through the inhibition of histone acetylation ${ }^{43,44}$ and the recruitment of DNA methyltransferase (DNMT) proteins ${ }^{45}$. ChIP assays were performed using antibodies against H1.2 and H1.5, which are replicationdependently expressed subtypes, and H1.0 and H1X, which are replication-independently expressed subtypes ${ }^{42}$. Although the amount of histone H1.2 around the TERT TSS in TAF-I KD cells was slightly higher than that in WT cells (Fig. 6E), this difference was not remarkable compared with that observed in active histone markers (Fig. 5A,B). Furthermore, the amount of histone H1.0, H1.5, and H1X on the TERT TSS was not significantly different among WT and TAF-I KD cells (Fig. 6F). These results suggest that flux of histone H1s on the TERT TSS may not be required for the transcriptional regulation of TERT by TAF-I.

Transient expression of exogenous TAF-I in TAF-I KD cells partially rescued the TERT transcription and induced the local reduction of CpG methylation at CGI-1 of TERT. To get more insight about how TAF-I is involved in TERT transcription through the changes of epigenetic marks, we performed the rescue experiments by transiently expressing shRNA-resistant TAF-I (resTAF-I) in TAF-I KD cells. In our experimental condition, we just succeeded to express a few (less than $50 \%$ of endogenous TAF-I) amounts of resTAF-I $\alpha$ and resTAF-I $\beta$ in TAF-I KD cells (Fig. 7A, and Supplementary Fig. $1 \mathrm{H}-\mathrm{M}$ ). The amount of TERT mRNA in resTAF-I-expressed cells were approximately 3.5-fold increased compared to that in empty vector DNA-transfected cells, while its expression level does not reach that in WT \#7 cells (Fig. 7B). We also found that the level of histone $\mathrm{H} 3 \mathrm{~K} 9 \mathrm{~K} 14 \mathrm{ac}$ was approximately 1.3-fold increased in resTAF-I-expressed cells compared to that in empty vector DNA-transfected cells, although the level of histone H3 K4me3 was not significantly increased (Fig. 7C). We also found that the CpG methylation level around nucleotide positions -90 to -100 at CGI-1 of TERT was reduced by transient expression of resTAF-I in TAF-I KD cells (Fig. 7D, indicated by red arrows). It is possible that TAF-I initially induces the reduction of CpG methylation around nucleotide positions -90 to -100 at CGI- 1 and the increase of histone H3 acetylation around TSS to upregulate TERT transcription from the epigenetically transcription-repressed state.

Down-regulation of TERT transcription by TAF-I KD was observed in not only HeLa cells but also other cancer cells. HeLa cells, an HPV-18-positive cervical cancer cell line, are well studied to clarify the regulatory mechanisms of TERT gene expression. Although we found that TAF-I is involved in transcriptional activation of TERT in HeLa cells, whether it is specific for HeLa cells or general for other cancer cells is unclear. To answer this question, we tried to establish TAF-I KD cell lines using other cancer cells. We succeeded to obtain stable TAF-I KD cell lines using A549 cells (a lung cancer) and HCT116 cells (a colorectal cancer). In TAF-I KD A549 cells and HCT116 cells, the expression level of TAF-I $\alpha$ and TAF-I $\beta$ were reduced to approximately $25 \%$ or less than $25 \%$ of that in control cells, respectively (Fig. $8 \mathrm{~A}, \mathrm{~B}$, and Supplementary Fig. $1 \mathrm{~N}-\mathrm{R}$ ). In both TAF-I KD A549 and HCT116 cells, the amount of TERT mRNA was reduced to approximately $30 \%$ of that in control cells, suggesting that TAF-I is involved in the TERT transcription in not only HeLa cells but also A549 and HCT116 cells (Fig. 8C,D). To examine whether TAF-I is involved in the epigenetic changes around TERT TSS in A549 and HCT116 cells, we performed bisulfite sequencing analyses to evaluate the level of CpG methylation at CGI-1 of TERT. In contrast to HeLa cells, CpG dinucleotides at CGI-1 of TERT were almost entirely unmethylated in both A549 and HCT116 control cells (Fig. 8E,F). However, the level of CpG methylation at CGI-1 of TERT were entirely increased in both TAF-I KD A549 and HCT116 cells by TAF-I KD (Fig. 8E,F). 

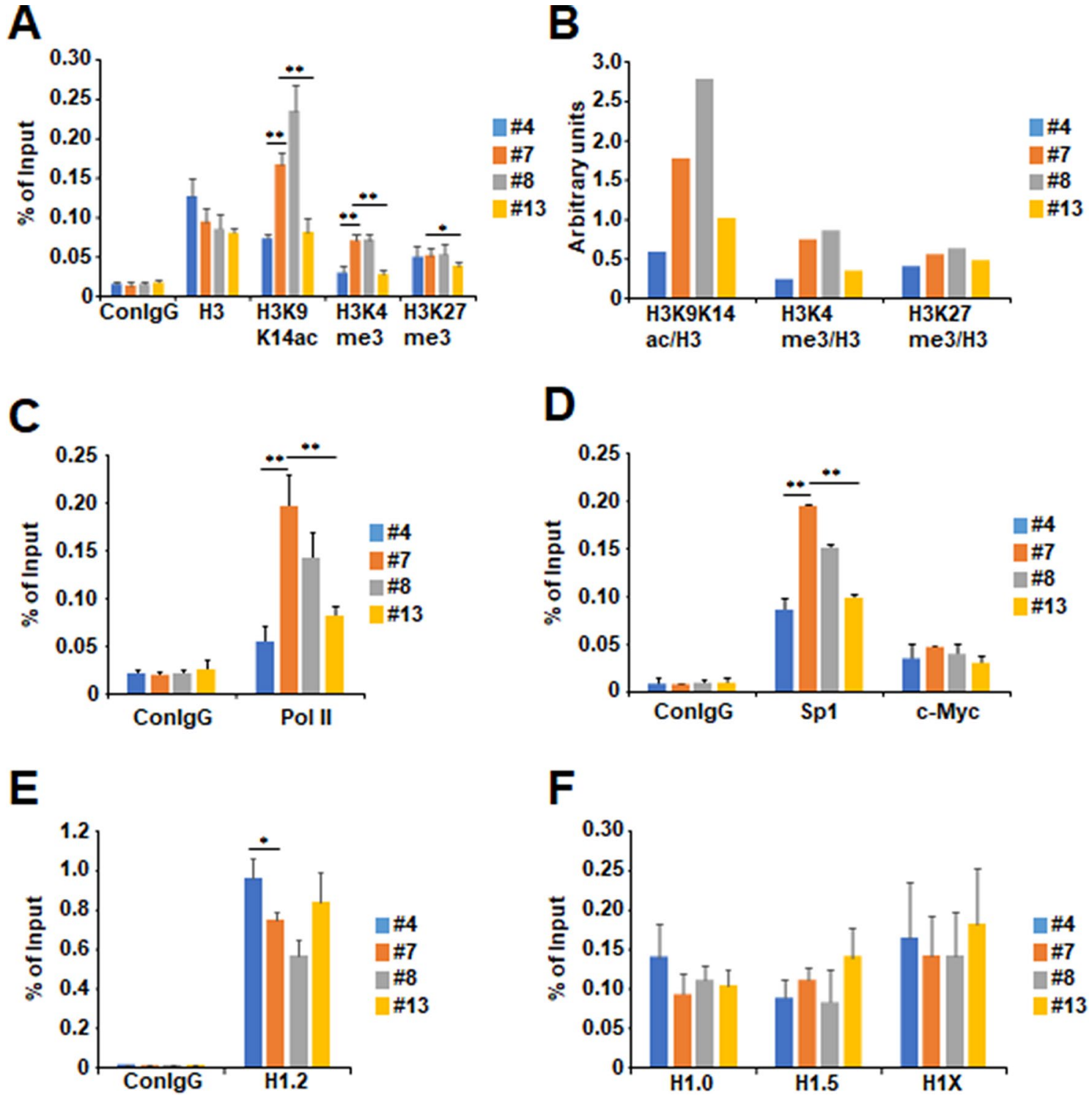

Figure 6. Histone modifications and $\mathrm{Sp} 1$ involved in transcriptional activation on TERT promoter were decreased in TAF-I KD HeLa cell lines independently of histone H1 amounts. ChIP assays were performed using WT and TAF-I KD HeLa cell lines. (A) The level of histone H3, H3 acetylated K9 and K14 (H3K9K14ac), $\mathrm{H} 3$ trimethylated $\mathrm{K} 4(\mathrm{H} 3 \mathrm{~K} 4 \mathrm{me} 3)$, and $\mathrm{H} 3$ trimethylated $\mathrm{K} 27$ (H3K27me3) around the TERT TSS including core promoter was examined. Rabbit normal IgG was used as a control for monitoring background (ConIgG). Data were represented as $\%$ of input at vertical line. Values represent the mean $\pm \mathrm{SD}(n=3)$. ${ }^{\star \star} P<0.01$ and ${ }^{\star} P<0.05$ relative to \#7. (B) The value of each histone $\mathrm{H} 3$ modification was normalized by that of histone $\mathrm{H} 3$. Arbitrarily units were represented at vertical line. (C,D) The level of rabbit normal IgG (conIgG), RNA polymerase II (pol II), Sp1, and c-Myc around the TERT TSS including core promoter was examined. Values represent the mean $\pm \mathrm{SD}(\mathrm{n}=3) .{ }^{*} P<0.01$ relative to \#7. (E,F) The level of rabbit normal IgG, histone H1.2, H1.0, H1.5, and H1X around the TERT TSS including core promoter was examined. Due to the difference of scale of vertical line, data were separately represented for histone H1.2 (C) and other histone H1s (D). Values represent the mean $\pm \mathrm{SD}(\mathrm{n}=3) .{ }^{\star} \mathrm{P}<0.05$ relative to $\# 7$.

Taken together, these results suggest that TAF-I is generally involved in TERT transcription through the alterations of CPG methylation around TERT TSS in several kinds of cancer cells.

\section{Discussion}

In this study, we found that TAF-I is a novel regulator of telomere DNA synthesis through the transcriptional activation of TERT through epigenetic mechanism in human cancer cells. TAF-I maintains the histone $\mathrm{H} 3$ modifications involved in transcriptional activation and hypomethylated cytosines in CpG dinucleotides around the TSS including minimal core promoter in the TERT.

Because a variety of cancer cells acquire the reactivation of telomerase, the transcriptional activation of TERT is a crucial step for occurrence and recurrence of human cancer cells. It is well known that several nucleotide 
A

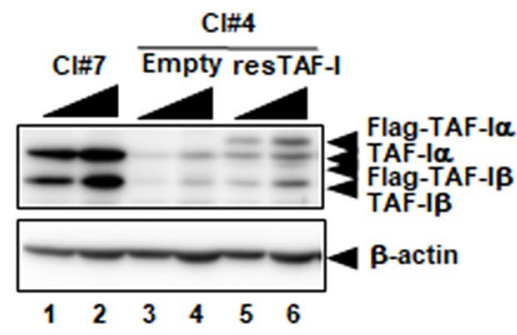

B

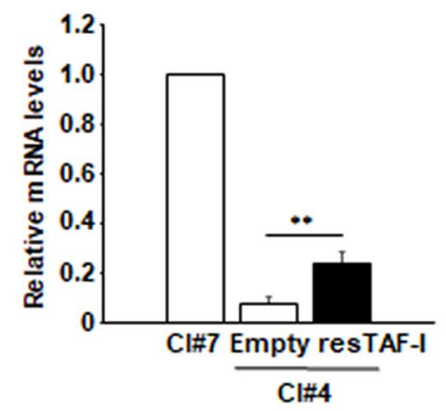

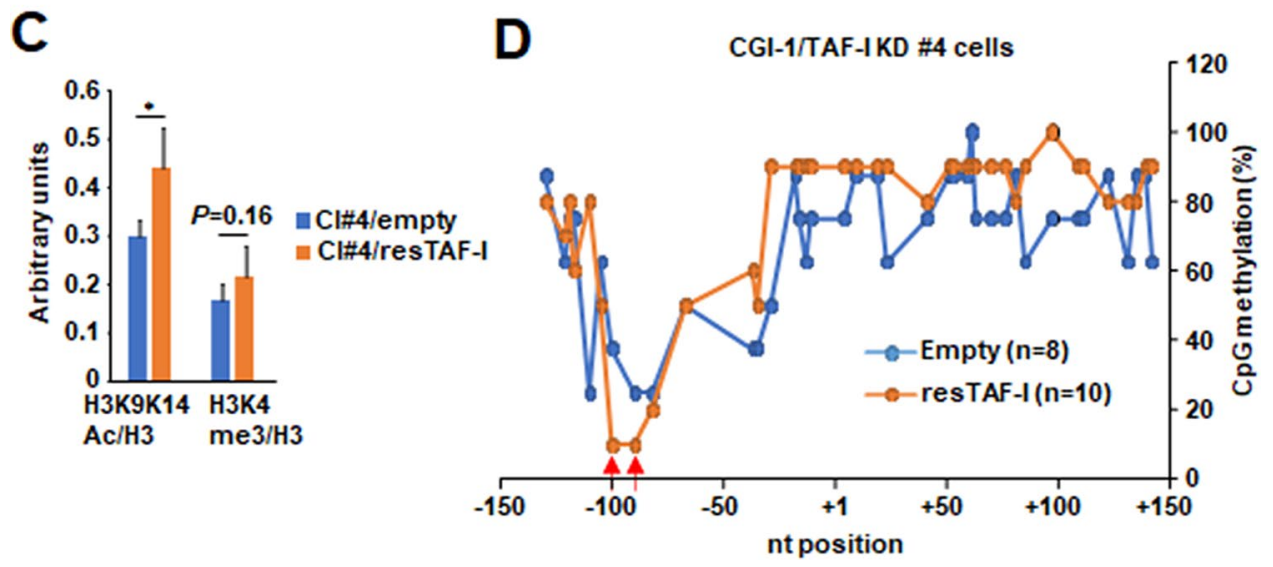

Figure 7. Transient expression of TAF-I partially rescued TERT transcription in TAF-I KD cells. (A) TAF-Ia and TAF-I $\beta$ expression levels were examined by western blotting analyses in TAF-I KD clone \#4 cells transfected with plasmid DNAs expressing shRNA-resistant Flag-TAF-I $\alpha$ and Flag-TAF-I $\beta$ (resTAF-I). Total cell extracts prepared from WT clone \#7 cells (lanes 1-2), and TAF-I KD clone \#4 cells transfected with empty vector DNA (lanes 3-4) or vector DNAs expressing resTAF-I (lane 5-6) were separated on 10\% SDS-PAGE followed by western blotting analyses using anti-TAF-I $\alpha / \beta$ and anti- $\beta$-actin antibodies. Ten (lanes 1,3 , and 5 ), and twenty (lanes 2, 4, and 6) $\mu \mathrm{g}$ of total protein prepared from each cell line was loaded. (B) The expression level of TERT mRNA was examined. Total RNA was isolated from each cell, and were subjected to Q-RT-PCR analyses to examine the expression level of TERT mRNA. The $18 \mathrm{~S}$ rRNA was used for normalization as an internal control. Values represent the mean $\pm S D(n=3) .{ }^{*} P<0.01$ relative to $\# 4$ transfected with empty vector DNA. (C) The level of histone $\mathrm{H} 3, \mathrm{H} 3 \mathrm{~K} 9 \mathrm{~K} 14 \mathrm{ac}$, and $\mathrm{H} 3 \mathrm{~K} 4 \mathrm{me} 3$ around the TERT TSS including core promoter was examined by ChIP assay. The value of each histone $\mathrm{H} 3$ modification was normalized by that of histone $\mathrm{H} 3$. Arbitrarily units were represented at vertical line. Values represent the mean $\pm S D(n=3) .{ }^{\star} P<0.01$. (D) Bisulfite sequencing analyses. The methylation level of each CpG dinucleotide in CGI-1 of the TERT locus was compared between TAF-I KD clone \#4 cells transfected with empty vector DNA and vector DNAs expressing resTAF-I. The horizontal line indicates nucleotide positions from the TERT TSS, and the vertical line indicates the percentage of CpG methylation. Two CpG nucleotides we observed reduction of methylation level by expression of resTAF-I were indicated by red arrows.

mutations in the TERT promoter such as C228T and C250T, both generating new binding sites for ETS family transcription factors, are frequently observed in human cancer cell lines. In addition to these genetic changes, epigenetic changes such as DNA methylation of CpG dinucleotides and histone modifications are also involved in the activation of TERT transcription. We found that TAF-I KD enhanced the methylation level of CpG dinucleotides around the TSS including minimal core promoter, but not the upstream region overlapping to THOR, in the TERT locus in HeLa cells. Furthermore, we observed that TAF-I KD simultaneously induces the reduction of TERT mRNA and upregulation of CpG methylation at CGI-1 of TERT in A549 and HCT116 cells. Therefore, it is possible that TAF-I is involved in the transcriptional activation of TERT through the maintenance of low CpG methylation level around TSS but not THOR in a variety of cancer cells. A previous report showed the dual-methylation pattern at the TERT promoter, in which the THOR is hypermethylated while the minimal promoter including C228T and C250T sites is hypomethylated in thyroid cancer cell lines ${ }^{16}$. Such dual-methylation pattern was also reported in other study ${ }^{18}$. These studies raise a possibility that the CpG methylation in the TERT locus is separately regulated at the THOR and the minimal promoter, and both regulations are important for the reactivation of TERT in cancer cells. Our findings become a clue to clarify how the locus-specific CpG methylation is regulated in the TERT locus. Interestingly, the methylation level at nucleotide position -67 from TSS, juxtaposed to the C228 nucleotide, showed the most marked difference between WT and TAF-I KD cells 
A

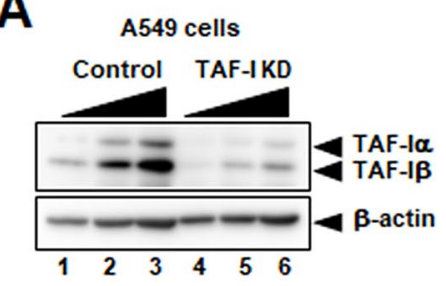

C

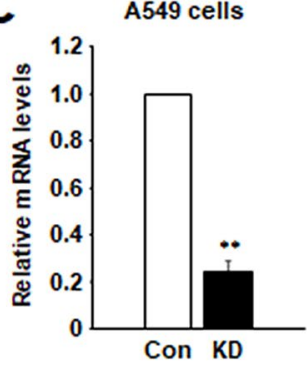

B

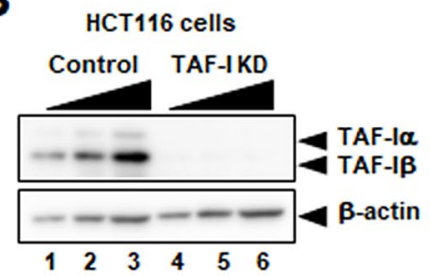

D

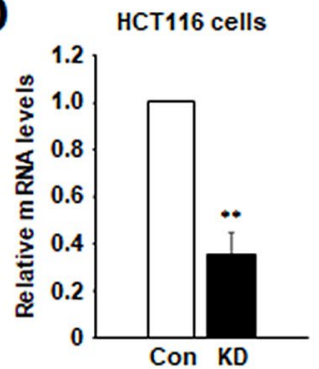

$\mathbf{F}$
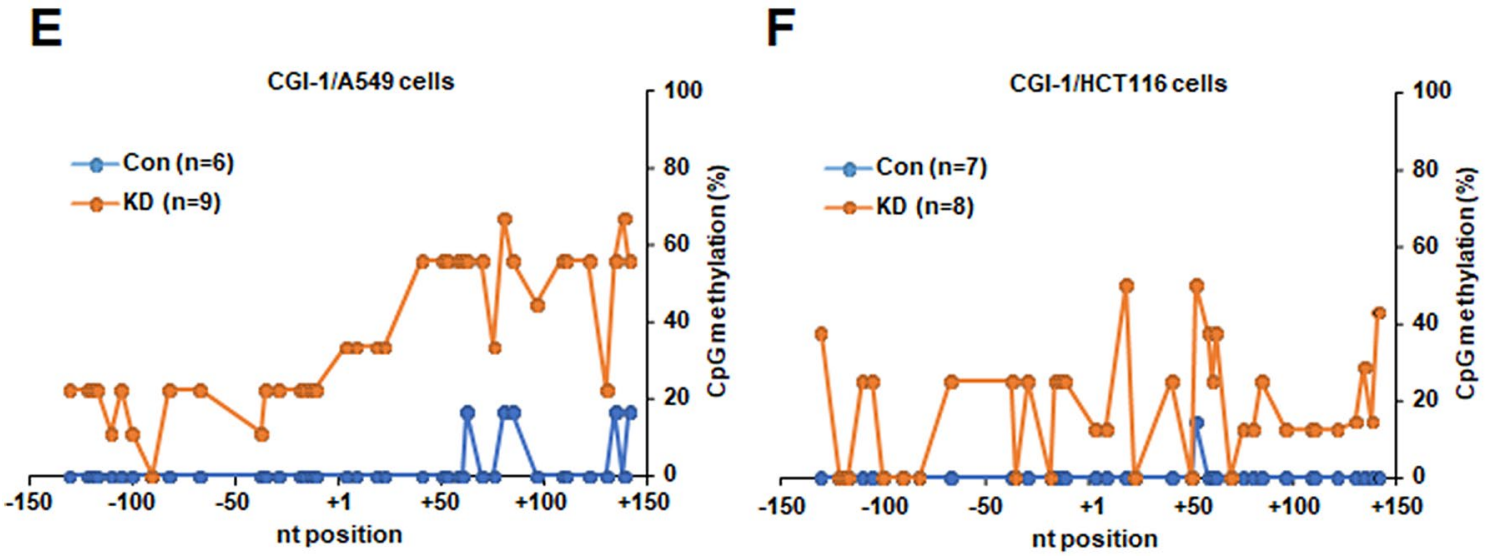

Figure 8. Downregulation of TERT transcription by TAF-I KD was observed in several cancer cells. $(\mathbf{A}, \mathbf{B})$ TAF-I $\alpha$ and TAF-I $\beta$ expression levels were examined by western blotting analyses. Total cell extracts prepared from control (lanes 1-3) and TAF-I KD (lanes 4-6) A549 cell lines (A), control (lanes 1-3) and TAF-I KD (lanes 4-6) HCT116 cell lines (B) were separated on 10\% SDS-PAGE followed by western blotting analyses using antiTAF-I $\alpha / \beta$ and anti- $\beta$-actin antibodies. Five (lanes 1 and 4), ten (lanes 2 and 5) and twenty (lanes 3 and 6 ) $\mu \mathrm{g}$ of total protein prepared from each cell line was loaded. (C,D) The expression level of TERT mRNA was examined. Total RNA was isolated from control (Con) and TAF-I KD (KD) A549 cells (C) or HCT116 cells (D), and were subjected to Q-RT-PCR analyses to examine the expression level of TERT mRNA. The $18 \mathrm{~S}$ rRNA was used for normalization as an internal control. Values represent the mean $\pm \mathrm{SD}(\mathrm{n}=3) .{ }^{* *} P<0.01$ relative to respective control cells. (E,F) Bisulfite sequencing analyses. The methylation level of each CpG dinucleotide in CGI-1 of TERT was compared between control and TAF-I KD A549 cells (E) or HCT116 cells (F). The horizontal line indicates nucleotide positions from the TERT TSS, and the vertical line indicates the percentage of CpG methylation.

(WT is approximately $30 \%$, and $\mathrm{KD}$ is approximately $95 \%$ in Fig. 4A,B, indicated in a red arrow). It is possible that the maintenance of low CpG dinucleotide methylation around C228 nucleotide is also important for the upregulation of the TERT transcription. In this study, we used HeLa cells, a human cervical cancer cell line, and observed neither C228T nor C250T mutations in genomic DNA, suggesting that these genetic changes do not occur and contribute to the TERT transcription in HeLa cells. However, it is possible that TAF-I-mediated maintenance of low CpG methylation cooperatively facilitates the reactivation of telomerase in combination with C228T mutation by assisting efficient binding of an ETS family transcription factor to the TERT promoter. Furthermore, we observed that transient expression of exogenous TAF-I partially rescues the TERT transcription in concert with the reduction of CpG methylation around nucleotide positions - 90 to - 100 at CGI-1 of TERT in TAF-I KD HeLa cells. Because the DNA around nucleotide positions - 90 to - 100 contains several Sp1 binding sites and also a C250 nucleotide (Fig. 4A), it is probable that this DNA region has an important role in TERT transcription. It is possible that TAF-I initially acts on DNA around nucleotide positions - 90 to -100 at CGI-1 to change the TERT to the epigenetically transcription-activated state. We also observed that the levels of histone H3 K9K14ac and K4me3 are also reduced at the TERT TSS in TAF-I KD cells in along with increase of $\mathrm{CpG}$ methylation, although the changes of histone modification level seemed to be not completely correlated with that of TERT mRNA. It is likely that transcription of TERT is cooperatively regulated by many 
factors including histone modifications, DNA methylations, and transcription factors. We observed that not only the level of histone modifications but also the level of CpG methylation and promoter-bound Sp1 are also regulated by TAF-I KD. Thus, the level of histone modifications may be not simply correlated with the level of TERT transcription. These results suggest that TAF-I is involved in transcriptional activation of TERT through the maintenance of multiple epigenetic marks generally involved in the transcriptional activation. Sp1 may be one of key factors involved in TAF-I-dependent epigenetic changes around TERT TSS.

TAF-I is a multifunctional protein involved in many cellular processes including transcription ${ }^{28,29}$, DNA repair $^{46}, \mathrm{M}$-phase chromosome dynamics ${ }^{47}$, cell migration ${ }^{48}$, apoptosis ${ }^{49}$, and so on. As a clinical significance of TAF-I, TAF-I $\beta$ but not TAF-I $\alpha$ was identified as SET which consists of a part of translocated gene found in an acute leukemia patient ${ }^{50}$. Several reports suggested that expression level of TAF-I is increased and implicated in poor clinical outcomes in many types of cancer cells ${ }^{51-56}$. These reports suggest that TAF-I possibly has an oncogenic activity, however its detailed function in cancer progression is not completely understood. TAF-I suppresses the $\mathrm{p} 53$-mediated transcription through the inhibition of its acetylation like an INHAT ${ }^{57}$. Furthermore, it is reported that inhibition of PP2A by TAF-I is important for tumorigenesis in several cancer cells. In addition to these observations, this study proposes that TAF-I is involved in the occurrence and/or recurrence of cancer cells through the upregulation of TERT transcription as a novel epigenetic regulator. In this study, we did not clarify the detailed mechanisms how TAF-I specifically regulates epigenetic marks around the TSS in the TERT locus. It is reported that TAF-I functions as a transcriptional repressor in several genes by inhibiting acetylation of histone H3, although we observed the opposite effect on the TERT. It is possible that TAF-I subtilizes its functions of epigenetic regulation dependently on what genes are, where genomic loci are, and which type of cells are. It is difficult to directly identify which cis- and trans-acting factors cooperate with TAF-I in the TERT transcription due to its multifunctionality, but the methylome analyses to observe where genomic loci are affected by TAF-I may clarify the common pattern of gene structure under the control of TAF-I similar to the TERT locus.

\section{Materials and methods}

Cell cultures, antibodies, and chemicals. All WT and TAF-I KD HeLa cell lines were cloned by picking up well-isolated drug-resistant colonies using cloning rings ${ }^{33}$. TAF-I KD HeLa cell lines, HEK293T cells, T98G cells, A549 cells, and HCT116 cells were maintained in Dulbecco modified Eagle medium (DMEM, Nissui) containing $10 \%$ FBS. Antibodies used in this study were as follows: anti-TAF-I $\alpha / \beta$ antibody (monoclonal antibody KM1720; Kirin-Kyowa Hakko), anti- $\beta$-actin, and anti-Sp1 antibodies from SIGMA; anti-histone H3, antihistone H3 K4me3, anti-histone H3 K27me3, anti-pol II (8WG16), anti-histone H1.2, anti-histone H1.5, and anti-histone H1X antibodies from Abcam; anti-c-Myc antibody from CST; normal rabbit IgG, and anti-histone H3 K9K14ac antibodies from Millipore; anti-histone H1.0 antibody from ProteinTech Group, Inc. Actionomycin D was purchased from SIGMA. Decitabine was purchased from BioVision.

Reverse transcription and quantitative PCR analyses. Total RNA was prepared from cells by acid guanidinium thiocyanate-phenol chloroform extraction (AGPC) method. The concentration of RNA in each sample was determined using a Nano Drop Lite spectrophotometer (Thermo Scientific). To analyze the level of the TERT mRNA and 18S rRNA, cDNA was synthesized from the total RNA prepared from HeLa cell lines using ReverTra Ace reverse transcriptase (TOYOBO) and a 9-mer random primer. The primer sets used for quantitative PCR (Q-PCR) were as follows: for TERT, 5'-CACGCGAAAACCTTCCTCAG-3' and 5'-TGTTCC TCCCAGCCTTGAAG-3'; TERC, 5'-GCTGTTTTTCTCGCTGACTTTCA-3' and 5'-GCAGCTGACATTTTT TGTTTGC-3'; TET1, 5'-TCCTGGTGCTATTCCAGTCC-3' and 5'-CAGGAAGGAAGACAGGCAAG-3'; for 18S rRNA, 5'-AACGGCTACCACATCCAAGG-3' and 5'-GGGAGTGGGTAATTTGCGC-3'. Q-PCR reactions were performed with FastStart SYBR Green Master (Roche) using Thermal Cycler Dice (TaKaRa). For the investigation of the TERT mRNA stability, $1 \mu \mathrm{M}$ of actinomycin D was added to cell culture medium and incubated cells for 1, 2, 4 and $8 \mathrm{~h}$. After collecting cells, total RNA was purified and subjected to reverse transcription and Q-PCR analyses as mentioned above.

Telomere repeat amplification protocol (TRAP) assay and Q-TRAP assay. TRAP assay was basically performed as previously described ${ }^{58}$. Each cell line was collected by trypsinization and subjected to counting of cell numbers. Same number of each cell treated with ice-cold hypotonic buffer ( $10 \mathrm{mM}$ Hepes- $\mathrm{NaOH}$ [pH7.5], $10 \mathrm{mM} \mathrm{KCl}, 1.5 \mathrm{mM} \mathrm{MgCl}$ and $1 \mathrm{mM} \mathrm{DTT}$ ) was centrifuged, then supernatant was discarded. Cells further incubated with lysis buffer (10 mM Tris- $\mathrm{HCl}$ [pH7.4], $1 \mathrm{mM} \mathrm{MgCl}, 1 \mathrm{mM}$ EGTA, 0.5\% CHAPS, $0.1 \mathrm{mM}$ PMSF and $5 \mathrm{mM} \beta$-mercaptoethanol) on ice for $30 \mathrm{~min}$ were subjected to centrifugation, then supernatant was collected and stored in $-80^{\circ} \mathrm{C}$ until use. Cell lysate prepared from desired number of cells was mixed with ACX primer, RNase inhibitor, KOD-Plus-PCR enzyme (TOYOBO), and with or without TS primer in PCR reaction buffer for KOD-Plus and incubated at $30^{\circ} \mathrm{C}$ for $30 \mathrm{~min}$, then subjected to PCR. When required, cell lysate was pre-incubated with RNaseA or heat-denatured at $95^{\circ} \mathrm{C}$ for $30 \mathrm{~min}$. PCR was performed with 26 cycles of $96^{\circ} \mathrm{C}$ for $20 \mathrm{~s}$ and $60^{\circ} \mathrm{C}$ for $1 \mathrm{~min}$. PCR products were separated on $8 \%$ polyacrylamide gel electrophoresis in $1 \times \mathrm{TBE}$ buffer, and subjected to EtBr staining. The primers used for TRAP assay were as follows: TS primer, 5'-AAT CCGTCGAGCAGAGTT-3'; ACX primer, 5'-GCGCGGCTTACCCTTACCCTTACCCTAACC-3'. For Q-TRAP assay, same cell lysates used in TRAP assay were mixed with ACX primer, RNase inhibitor, TS primer, and KODPlus- SYBR mix (TOYOBO). Mixtures were incubated at $30{ }^{\circ} \mathrm{C}$ for $30 \mathrm{~min}$, then directly subjected to Q-PCR analyses using Thermal Cycler Dice.

Telomere-FISH assay. Telomere-FISH assay was basically performed as previously described with several modifications ${ }^{34}$. Each HeLa cell line grown on glass cover slip was washed with PBS (-) and fixed with $4 \%$ 
formaldehyde at room temperature for $10 \mathrm{~min}$. Fixed cells on coverslip were briefly washed with $0.2 \times \mathrm{SSC}$, then dehydrated with 70,85 , and $90 \% \mathrm{EtOH}$ in a stepwise fashion at room temperature for $3 \mathrm{~min}$, respectively. After cells were incubated with $10 \mathrm{mM}$ sodium citrate (pH6.5) at $85^{\circ} \mathrm{C}$ for $10 \mathrm{~min}$, cells were washed with PBS (-) and dipped through 25,50 and $95 \% \mathrm{EtOH}$ in a stepwise fashion at room temperature for $3 \mathrm{~min}$, respectively. Cells were incubated with PBS (-) containing $0.2 \mathrm{mg} / \mathrm{ml} \mathrm{RNase} \mathrm{A}$ at $37^{\circ} \mathrm{C}$ for $10 \mathrm{~min}$, washed with PBS (-) and again dipped through 25, 50 and $95 \% \mathrm{EtOH}$ in a stepwise fashion at room temperature for $3 \mathrm{~min}$, respectively. Cells were incubated with hybridization buffer $(10 \mathrm{mM}$ Tris- $\mathrm{HCl}$ [pH7.4], 70\% formamide, and 1xBlocking reagent [Roche]) containing $100 \mathrm{nM}$ TelC-Cy3 PNA probe (Panagene) at $86^{\circ} \mathrm{C}$ for $6 \mathrm{~min}$, and further incubated at room temperature for $2.5 \mathrm{~h}$ in the dark. Cells were washed with hybridization buffer at room temperature for $15 \mathrm{~min}$, followed by PBS (-) containing $0.05 \%$ Tween 20 at room temperature for $5 \mathrm{~min}$. Finally, DNA was stained with DAPI, then coverslips were sealed on slide glass and subjected to observation using LSM700 confocal microscope. Data analyses were performed by Image J Fiji software.

Terminal restriction fragment (TRF) assay. Each collected HeLa cell line was treated with ProteinaseK, and whole nucleic acids were purified by phenol/ $\mathrm{CHCl}_{3}$ extraction and $\mathrm{EtOH}$ precipitation. Whole nucleic acids were treated with $\mathrm{RNaseA}$, then again subjected to phenol/ $\mathrm{CHCl}_{3}$ extraction and $\mathrm{EtOH}$ purification to purify genomic DNA. After each genomic DNA was digested with HinfI and RsaI, DNA were purified by phenol/ $\mathrm{CHCl}_{3}$ extraction and EtOH purification. Ten $\mu \mathrm{g}$ of each DNA was separated by $0.8 \%$ agarose gel electrophoresis in $1 \times \mathrm{TBE}$ buffer at $100 \mathrm{~V}$ constant for $4.5 \mathrm{~h}$, DNA was visualized by staining with GelRed, then transferred onto Biodyne nylon membrane (PALL) by alkali transfer method. After membrane was pre-hybridized with hybridization buffer ( $5 \times \mathrm{SSC}, 5 \times$ Denhardt's solution, $0.5 \mathrm{mM}$ tetrasodium pyrophosphate, $10 \mathrm{mM} \mathrm{Na}_{2} \mathrm{HPO}_{4}, 0.1 \%$ SDS, and $0.1 \mathrm{mg} / \mathrm{ml}$ sermon sperm DNA) at $37^{\circ} \mathrm{C}$ for $1 \mathrm{~h},{ }^{32} \mathrm{P}$-labeled telomere probe was added and further incubated at $37^{\circ} \mathrm{C}$ for overnight. After membrane was washed for three times with wash buffer $(0.1 \times$ SSC and $0.1 \%$ SDS) at $22^{\circ} \mathrm{C}$ for $7 \mathrm{~min}$, images were taken by Typhoon FLA 7000. The telomere probe DNA was as follows: $5^{\prime}$-CCCTTACCCTTACCCTTA-3'. Telomere probe DNA was radiolabeled with $\left[\gamma^{-32} \mathrm{P}\right]$ ATP by incubation with T4 polynucleotide kinase. The telomere length of each sample was evaluated using WALTER, an online toolset ${ }^{59}$.

Cell growth assay. Cell growth was evaluated by WST- 8 assay using CCK- 8 reagents (DOJINDO). WT and TAF-I KD HeLa cells $\left(5 \times 10^{3}\right.$ cells $)$ were plated onto 96 well plates with $100 \mu \mathrm{L}$ DMEM-10\% FBS. After incubation for 24,48 , and $72 \mathrm{~h}, 100 \mu \mathrm{L}$ of CCK-8 reagents was added to medium and incubated at $37^{\circ} \mathrm{C}$ for $1 \mathrm{~h}$. Absorbance at $450 \mathrm{~nm}$ was measured by microplate reader (TECAN).

Plasmid transfection and luciferase assay. A series of TERT promoter-fused firefly luciferase reporter plasmid DNA (pTERT-Luc) was previously established ${ }^{39}$. HeLa cell lines were transfected with pTERT-Luc plasmid DNAs using GeneJuice (Novagen) in combination with a control plasmid DNA pEF1a-RLuc expressing renilla luciferase. At $48 \mathrm{~h}$ after transfection, cells were washed with PBS (-) and lysed in a cell lysis buffer for renilla luciferase (Promega) by three freezing-thawing cycles. The cell lysates and a firefly or renilla luciferase substrate (Promega) were mixed, and both luciferase activities were measured by Lumat LB9506 (BERTHOLD). The firefly luciferase activities were normalized by the renilla luciferase activity.

Bisulfite sequencing analyses. Each collected HeLa, A549, and HCT116 cell line was treated with ProteinaseK, and whole nucleic acids were purified by phenol/ $\mathrm{CHCl}_{3}$ extraction and EtOH precipitation. Whole nucleic acids were treated with $\mathrm{RNaseA}$, then again subjected to phenol/ $\mathrm{CHCl}_{3}$ extraction and EtOH purification to purify genomic DNA. Each DNA concentration was measured by Nano Drop mini. Two $\mu \mathrm{g}$ of each genomic DNA was incubated in $0.3 \mathrm{M} \mathrm{NaOH}$ at $37^{\circ} \mathrm{C}$ for $15 \mathrm{~min}$, then $1.9 \mathrm{M}$ sodium bisulfite and $0.5 \mathrm{mM}$ hydroquinone were added and further incubated in PCR machine with 15 cycles of $30 \mathrm{~s}$ at $95^{\circ} \mathrm{C}$ and $15 \mathrm{~min}$ at $50^{\circ} \mathrm{C}$. DNAs were purified by EtOH precipitation, then subjected to PCR using TaKaRa EpiTaq ${ }^{\text {TM }} \mathrm{HS}^{\mathrm{aNA}} \mathrm{DN}$ polymerase (TaKaRa). The primers used for PCR were as follows: for the CGI-1, 5'-CGGGATCCTGTTTTGTTTTTTTAT TTTTTAG-3' and 5'-CGGGATCCCCAACCCTAAAACCCC-3'; for the CGI-2, 5'-CGGGATCCTTGTTTT TAGGGTTTTTATATTATGGT-3' and 5'-CGGGATCCCAAAACTAAAAAATAAAAAAACAAAAC-3'. PCR products were digested with BamHI and subcloned into BamHI-digested pcDNA3 plasmid DNA, then transformed into bacteria. Plasmid DNAs were isolated and subjected to sequencing reaction with BigDye terminator (ver3.1) using a primer corresponding to T7 promoter sequence.

Chromatin immunoprecipitation (ChIP) assay. Each HeLa cell line was fixed with 1\% formaldehyde by directly adding formalin into culture medium and incubated at room temperature for $10 \mathrm{~min}$. After adding $0.125 \mathrm{M}$ glycine and incubation at room temperature for $5 \mathrm{~min}$ to stop cross linking, cells were washed with PBS (-) and collected into tube by scraping and centrifugation. Cells were resuspended in lysis buffer (50 mM Tris-HCl [pH7.9], $10 \mathrm{mM}$ EDTA, 1\% SDS and $1 \mathrm{mM} \mathrm{PMSF)} \mathrm{and} \mathrm{subjected} \mathrm{to} \mathrm{lyse} \mathrm{by} \mathrm{sonication.} \mathrm{After} \mathrm{cen-}$ trifugation of cell suspensions, supernatants were collected and tenfold diluted with dilution buffer $(16.7 \mathrm{mM}$ Tris-HCl [pH7.9], $167 \mathrm{mM} \mathrm{NaCl}, 1.2 \mathrm{mM}$ EDTA, 1.1\% TritonX-100, 0.01\% SDS and 1.1 mM PMSF). Protein A-Sepharose beads pre-incubated with BSA and sermon sperm DNA were added to each lysate and rotate at $4{ }^{\circ} \mathrm{C}$ for $6 \mathrm{~h}$ to pre-clear the lysate. Supernatants were collected by centrifugation, each antibody was added and incubated at $4{ }^{\circ} \mathrm{C}$ for overnight with rotation. Protein A-Sepharose beads were added to lysate and further incubated at $4{ }^{\circ} \mathrm{C}$ for $1 \mathrm{~h}$. After beads were collected by centrifugation, washed with high salt wash buffer, $\mathrm{LiCl}$ wash buffer and TE in a stepwise fashion. DNA-protein complex bound with beads was eluted with elute buffer $\left(0.1 \mathrm{M} \mathrm{NaHCO}_{3}, 1 \% \mathrm{SDS}\right.$ and $\left.10 \mathrm{mM} \mathrm{DTT}\right)$. Elutes and a part of lysate were incubated at $65^{\circ} \mathrm{C}$ for $6 \mathrm{~h}$ to reverse cross-links, added with glycogen as a carrier, then DNAs were purified by phenol/ $\mathrm{CHCl}_{3}$ extraction and EtOH 
precipitation. Q-PCR reactions were performed with KOD-Plus- SYBR mix (TOYOBO) using Thermal Cycler Dice (Takara). The value of DNA derived from lysate (input) was used for normalization to calculate the $\%$ of input. The primers used for PCR were as follows: for the TERT promoter TSS, $5^{\prime}$-AGCCCCTCCCCTTCCTTT CC-3' and 5'-AGCGCACGGCTCGGCAGC-3'.

Rescue experiments by expression of Flag-TAF-I in TAF-I KD HeLa cells. TAF-I KD HeLa clone \#4 cells were transfected with pcDNA3.1(+)-Flag plasmid (empty vector DNA) or pcDNA3.1(+)-Flag-TAF-Ia and pcDNA3.1(+)-Flag-TAF-I $\beta$ plasmids ${ }^{28}$ expressing short hairpin RNA-resistant TAF-I $\alpha$ and TAF-I $\beta$ using GeneJuice in combination with pBabe-puro plasmid expressing puromycin-resistant gene. At $24 \mathrm{~h}$ after transfection, $2 \mu \mathrm{g} / \mathrm{mL}$ puromycin was directly added to medium and cells were further incubated for $24 \mathrm{~h}$. After medium including dead cells were removed from dishes, cells were rinsed with PBS (-), then fresh medium was added and further incubated for $48 \mathrm{~h}$. Cells were harvested and subjected to western blotting, total RNA purification following Q-RT-PCR, ChIP assay, and bisulfite sequencing analyses.

Establishment of stable TAF-I KD cell lines in A549 and HCT116 cells. A549 and HCT116 cells were transfected with pU6i-puro-shEGFP or pU6i-puro-shTAF-I plasmids ${ }^{28}$ using GeneJuice. At $24 \mathrm{~h}$ after transfection, $2 \mu \mathrm{g} / \mathrm{mL}$ of puromycin was added to cell culture medium and further incubated for 24 to $36 \mathrm{~h}$. After medium including dead cells were removed from dishes, cells were rinsed with PBS (-), fresh medium was added and further incubated for $12 \mathrm{~h}$. Cells were once replated onto $\phi 10 \mathrm{~cm}$ dishes with medium and maintain until cell colonies grow to appropriate size. Multiple well-isolated colonies were picked up by trypsinization using cloning rings, transferred into fresh medium, and maintained until acquiring enough numbers of cells. Western blotting analyses using anti-TAF-I antibody were performed with obtained each cell clone. One of cell lines transfected with pU6i-puro-shEGFP which maintain the expression level of TAF-I similar to that in parental A549 or HCT116 cells was defined as control cells. Cell lines in which expression level of TAF-I is efficiently reduced compared to that in control cells were defined as TAF-I KD cells.

Received: 5 September 2020; Accepted: 19 August 2021

Published online: 06 September 2021

\section{References}

1. Olovnikov, A. M. A Theory of Marginotomy: The incomplete copying of template margin in enzymatic synthesis of polynucleotides and biological significance of the phenomenon. J. Theor. Biol. 41, 181-190. https://doi.org/10.1016/0022-5193(73)90198-7 (1973).

2. Hayflick, L. \& Moorhead, P. S. The serial cultivation of human diploid cell strains. Exp. Cell Res. 25, 585-621. https://doi.org/10. 1016/0014-4827(61)90192-6 (1961).

3. Cohen, S. B. et al. Protein composition of catalytically active human telomerase from immortal cells. Science 315, 1850-1853. https://doi.org/10.1126/science.1138596 (2007).

4. Gaspar, T. B. et al. Telomere maintenance mechanisms in cancer. Genes https://doi.org/10.3390/genes9050241 (2018).

5. Kyo, S. et al. Sp1 cooperates with c-Myc to activate transcription of the human telomerase reverse transcriptase gene (hTERT). Nucleic Acids Res. 28, 669-677. https://doi.org/10.1093/nar/28.3.669 (2000).

6. Oh, S., Song, Y., Yim, J. \& Kim, T. K. The Wilms' tumor 1 tumor suppressor gene represses transcription of the human telomerase reverse transcriptase gene. J. Biol. Chem. 274, 37473-37478. https://doi.org/10.1074/jbc.274.52.37473 (1999).

7. Renaud, S., Loukinov, D., Bosman, F. T., Lobanenkov, V. \& Benhattar, J. CTCF binds the proximal exonic region of hTERT and inhibits its transcription. Nucleic Acids Res. 33, 6850-6860. https://doi.org/10.1093/nar/gki989 (2005).

8. Huang, F. W. et al. Highly recurrent TERT promoter mutations in human melanoma. Science 339, 957-959. https://doi.org/10. $1126 /$ science. 1229259 (2013).

9. Horn, S. et al. TERT promoter mutations in familial and sporadic melanoma. Science 339, 959-961. https://doi.org/10.1126/scien ce.1230062 (2013).

10. Rachakonda, P. S. et al. TERT promoter mutations in bladder cancer affect patient survival and disease recurrence through modification by a common polymorphism. Proc. Natl. Acad. Sci. USA 110, 17426-17431. https://doi.org/10.1073/pnas.1310522110 (2013).

11. Cao, Y., Bryan, T. M. \& Reddel, R. R. Increased copy number of the TERT and TERC telomerase subunit genes in cancer cells. Cancer Sci. 99, 1092-1099. https://doi.org/10.1111/j.1349-7006.2008.00815.x (2008).

12. Barthel, F. P. et al. Systematic analysis of telomere length and somatic alterations in 31 cancer types. Nat. Genet. 49, 349-357. https://doi.org/10.1038/ng.3781 (2017).

13. Valentijn, L. J. et al. TERT rearrangements are frequent in neuroblastoma and identify aggressive tumors. Nat. Genet. 47, 1411-1414. https://doi.org/10.1038/ng.3438 (2015).

14. Peifer, M. et al. Telomerase activation by genomic rearrangements in high-risk neuroblastoma. Nature 526, 700-704. https://doi. org/10.1038/nature14980 (2015).

15. Stern, J. L. et al. Allele-specific DNA methylation and its interplay with repressive histone marks at promoter-mutant TERT genes. Cell Rep. 21, 3700-3707. https://doi.org/10.1016/j.celrep.2017.12.001 (2017).

16. Avin, B. A. et al. Characterization of human telomerase reverse transcriptase promoter methylation and transcription factor binding in differentiated thyroid cancer cell lines. Genes Chromosomes Cancer 58, 530-540. https://doi.org/10.1002/gcc.22735 (2019).

17. Lee, D. D., Komosa, M., Nunes, N. M. \& Tabori, U. DNA methylation of the TERT promoter and its impact on human cancer. Curr. Opin. Genet. Dev. 60, 17-24. https://doi.org/10.1016/j.gde.2020.02.003 (2020).

18. Lee, D. D. et al. DNA hypermethylation within TERT promoter upregulates TERT expression in cancer. J. Clin. Investig. 129, 223-229. https://doi.org/10.1172/JCI121303 (2019).

19. Ge, Z. et al. Chromatin remodeling: Recruitment of histone demethylase RBP2 by Mad1 for transcriptional repression of a Myc target gene, telomerase reverse transcriptase. FASEB J. 24, 579-586. https://doi.org/10.1096/fi.09-140087 (2010).

20. Zhang, B., Chen, J., Cheng, A. S. \& Ko, B. C. Depletion of sirtuin 1 (SIRT1) leads to epigenetic modifications of telomerase (TERT) gene in hepatocellular carcinoma cells. PLoS ONE 9, e84931. https://doi.org/10.1371/journal.pone.0084931 (2014). 
21. Stern, J. L., Theodorescu, D., Vogelstein, B., Papadopoulos, N. \& Cech, T. R. Mutation of the TERT promoter, switch to active chromatin, and monoallelic TERT expression in multiple cancers. Genes Dev. 29, 2219-2224. https://doi.org/10.1101/gad.269498. 115 (2015).

22. Matsumoto, K., Nagata, K., Ui, M. \& Hanaoka, F. Template activating factor I, a novel host factor required to stimulate the adenovirus core DNA replication. J. Biol. Chem. 268, 10582-10587 (1993).

23. Asaka, M. N., Murano, K. \& Nagata, K. Sp1-mediated transcription regulation of TAF-Ialpha gene encoding a histone chaperone. Biochem. Biophys. Res. Commun. 376, 665-670. https://doi.org/10.1016/j.bbrc.2008.09.053 (2008).

24. Okuwaki, M. \& Nagata, K. Template activating factor-I remodels the chromatin structure and stimulates transcription from the chromatin template. J. Biol. Chem. 273, 34511-34518. https://doi.org/10.1074/jbc.273.51.34511 (1998).

25. Miyaji-Yamaguchi, M., Okuwaki, M. \& Nagata, K. Coiled-coil structure-mediated dimerization of template activating factor-I is critical for its chromatin remodeling activity. J. Mol. Biol. 290, 547-557. https://doi.org/10.1006/jmbi.1999.2898 (1999).

26. Kato, K., Okuwaki, M. \& Nagata, K. Role of Template Activating Factor-I as a chaperone in linker histone dynamics. J. Cell Sci. 124, 3254-3265. https://doi.org/10.1242/jcs.083139 (2011).

27. Kajitani, K., Kato, K. \& Nagata, K. Histone H1 chaperone activity of TAF-I is regulated by its subtype-dependent intramolecular interaction. Genes Cells 22, 334-347. https://doi.org/10.1111/gtc.12478 (2017).

28. Kadota, S. \& Nagata, K. Silencing of IFN-stimulated gene transcription is regulated by histone $\mathrm{H} 1$ and its chaperone TAF-I. Nucleic Acids Res. 42, 7642-7653. https://doi.org/10.1093/nar/gku485 (2014).

29. Seo, S. B. et al. Regulation of histone acetylation and transcription by INHAT, a human cellular complex containing the set oncoprotein. Cell 104, 119-130. https://doi.org/10.1016/s0092-8674(01)00196-9 (2001).

30. Loven, M. A., Muster, N., Yates, J. R. \& Nardulli, A. M. A novel estrogen receptor alpha-associated protein, template-activating factor Ibeta, inhibits acetylation and transactivation. Mol. Endocrinol. 17, 67-78. https://doi.org/10.1210/me.2002-0280 (2003).

31. Almeida, L. O. et al. SET oncoprotein accumulation regulates transcription through DNA demethylation and histone hypoacetylation. Oncotarget 8, 26802-26818. https://doi.org/10.18632/oncotarget.15818 (2017).

32. Kato, K., Miyaji-Yamaguchi, M., Okuwaki, M. \& Nagata, K. Histone acetylation-independent transcription stimulation by a histone chaperone. Nucleic Acids Res. 35, 705-715. https://doi.org/10.1093/nar/gkl1077 (2007).

33. Haruki, H., Okuwaki, M., Miyagishi, M., Taira, K. \& Nagata, K. Involvement of template-activating factor I/SET in transcription of adenovirus early genes as a positive-acting factor. J. Virol. 80, 794-801. https://doi.org/10.1128/JVI.80.2.794-801.2006 (2006).

34. Sharifi-Sanjani, M., Meeker, A. K. \& Mourkioti, F. Evaluation of telomere length in human cardiac tissues using cardiac quantitative FISH. Nat. Protoc. 12, 1855-1870. https://doi.org/10.1038/nprot.2017.082 (2017).

35. Emerald, B. S. et al. AlphaCP1 mediates stabilization of hTERT mRNA by autocrine human growth hormone. J. Biol. Chem. 282, 680-690. https://doi.org/10.1074/jbc.M600224200 (2007).

36. Suzuki, T. et al. Functional interaction of the DNA-binding transcription factor Sp1 through its DNA-binding domain with the histone chaperone TAF-I. J. Biol. Chem. 278, 28758-28764. https://doi.org/10.1074/jbc.M302228200 (2003).

37. Miyamoto, S. et al. Positive and negative regulation of the cardiovascular transcription factor KLF5 by p300 and the oncogenic regulator SET through interaction and acetylation on the DNA-binding domain. Mol. Cell Biol. 23, 8528-8541. https://oi.org/ $10.1128 / \mathrm{mcb} .23 .23 .8528-8541.2003(2003)$.

38. Janghorban, M. et al. Targeting c-MYC by antagonizing PP2A inhibitors in breast cancer. Proc. Natl. Acad. Sci. USA 111, 9157-9162. https://doi.org/10.1073/pnas.1317630111 (2014).

39. Takakura, M. et al. Cloning of human telomerase catalytic subunit (hTERT) gene promoter and identification of proximal core promoter sequences essential for transcriptional activation in immortalized and cancer cells. Cancer Res. 59, 551-557 (1999).

40. Hebbar, P. B. \& Archer, T. K. Altered histone H1 stoichiometry and an absence of nucleosome positioning on transfected DNA. J. Biol. Chem. 283, 4595-4601. https://doi.org/10.1074/jbc.M709121200 (2008).

41. Allis, C. D. \& Jenuwein, T. The molecular hallmarks of epigenetic control. Nat. Rev. Genet. 17, 487-500. https://doi.org/10.1038/ nrg.2016.59 (2016).

42. Fyodorov, D. V., Zhou, B. R., Skoultchi, A. I. \& Bai, Y. Emerging roles of linker histones in regulating chromatin structure and function. Nat. Rev. Mol. Cell Biol. 19, 192-206. https://doi.org/10.1038/nrm.2017.94 (2018).

43. Herrera, J. E., West, K. L., Schiltz, R. L., Nakatani, Y. \& Bustin, M. Histone H1 is a specific repressor of core histone acetylation in chromatin. Mol. Cell Biol. 20, 523-529. https://doi.org/10.1128/mcb.20.2.523-529.2000 (2000).

44. Sun, J. et al. Histone H1-mediated epigenetic regulation controls germline stem cell self-renewal by modulating H4K16 acetylation. Nat. Commun. 6, 8856. https://doi.org/10.1038/ncomms9856 (2015).

45. Yang, S. M., Kim, B. J., NorwoodToro, L. \& Skoultchi, A. I. H1 linker histone promotes epigenetic silencing by regulating both DNA methylation and histone H3 methylation. Proc. Natl. Acad. Sci. USA 110, 1708-1713. https://doi.org/10.1073/pnas.12132 66110 (2013).

46. Kalousi, A. et al. The nuclear oncogene SET controls DNA repair by KAP1 and HP1 retention to chromatin. Cell Rep. 11, 149-163. https://doi.org/10.1016/j.celrep.2015.03.005 (2015).

47. Asai, Y. et al. Aurora B kinase activity is regulated by SET/TAF1 on Sgo2 at the inner centromere. J. Cell Biol. 218, 3223-3236. https://doi.org/10.1083/jcb.201811060 (2019).

48. ten Klooster, J. P., Leeuwen, I., Scheres, N., Anthony, E. C. \& Hordijk, P. L. Rac1-induced cell migration requires membrane recruitment of the nuclear oncogene SET. EMBO J. 26, 336-345. https://doi.org/10.1038/sj.emboj.7601518 (2007).

49. Fan, Z., Beresford, P. J., Oh, D. Y., Zhang, D. \& Lieberman, J. Tumor suppressor NM23-H1 is a granzyme A-activated DNase during CTL-mediated apoptosis, and the nucleosome assembly protein SET is its inhibitor. Cell 112, 659-672. https://doi.org/10.1016/ s0092-8674(03)00150-8 (2003).

50. von Lindern, M. et al. Can, a putative oncogene associated with myeloid leukemogenesis, may be activated by fusion of its 3 ' half to different genes: Characterization of the set gene. Mol. Cell Biol. 12, 3346-3355. https://doi.org/10.1128/mcb.12.8.3346 (1992).

51. Neviani, P. et al. The tumor suppressor PP2A is functionally inactivated in blast crisis CML through the inhibitory activity of the BCR/ABL-regulated SET protein. Cancer Cell 8, 355-368. https://doi.org/10.1016/j.ccr.2005.10.015 (2005).

52. Christensen, D. J. et al. SET oncoprotein overexpression in B-cell chronic lymphocytic leukemia and non-Hodgkin lymphoma: A predictor of aggressive disease and a new treatment target. Blood 118, 4150-4158. https://doi.org/10.1182/blood-2011-04-351072 (2011).

53. Pippa, R. et al. MYC-dependent recruitment of RUNX1 and GATA2 on the SET oncogene promoter enhances PP2A inactivation in acute myeloid leukemia. Oncotarget 8, 53989-54003. https://doi.org/10.18632/oncotarget.9840 (2017).

54. Farrell, A. S. et al. Targeting inhibitors of the tumor suppressor PP2A for the treatment of pancreatic cancer. Mol Cancer Res 12, 924-939. https://doi.org/10.1158/1541-7786.MCR-13-0542 (2014).

55. Cristobal, I. et al. Deregulation of the PP2A inhibitor SET shows promising therapeutic implications and determines poor clinical outcome in patients with metastatic colorectal cancer. Clin. Cancer Res. 21, 347-356. https://doi.org/10.1158/1078-0432.CCR-140724 (2015).

56. Hung, M. H. et al. Upregulation of the oncoprotein SET determines poor clinical outcomes in hepatocellular carcinoma and shows therapeutic potential. Oncogene 35, 4891-4902. https://doi.org/10.1038/onc.2016.21 (2016).

57. Wang, D. et al. Acetylation-regulated interaction between p53 and SET reveals a widespread regulatory mode. Nature 538, 118-122. https://doi.org/10.1038/nature19759 (2016). 
58. Kim, N. W. \& Wu, F. Advances in quantification and characterization of telomerase activity by the telomeric repeat amplification protocol (TRAP). Nucleic Acids Res. 25, 2595-2597. https://doi.org/10.1093/nar/25.13.2595 (1997).

59. Lycka, M. et al. WALTER: An easy way to online evaluate telomere lengths from terminal restriction fragment analysis. $B M C$ Bioinform. 22, 145. https://doi.org/10.1186/s12859-021-04064-0 (2021).

\section{Acknowledgements}

We thank Dr. S. Kyo (Shimane University) for a series of pTERT-Luc reporter plasmid. This work was supported by Grants-in-Aid for Scientific Research from the Ministry of Education, Culture, Sports, Science, and Technology of Japan (18K06052 to K.K. and 19H03475 to A.K.), Japan Agency for Medical Research and Development (AGA02314 to AK), and the NOMURA Microbial Community Control Project of ERATO of the Japan Science and Technology Agency (to A.K.).

\section{Author contributions}

K.K. designed and performed experiments, prepared figures, and wrote the manuscript. A.K. advised for experiments and writing the manuscript. K.N. supervised whole work. All authors reviewed the manuscript.

\section{Competing interests}

The authors declare no competing interests.

\section{Additional information}

Supplementary Information The online version contains supplementary material available at https://doi.org/ 10.1038/s41598-021-97009-9.

Correspondence and requests for materials should be addressed to K.K. or K.N.

Reprints and permissions information is available at www.nature.com/reprints.

Publisher's note Springer Nature remains neutral with regard to jurisdictional claims in published maps and institutional affiliations.

(c) (i) Open Access This article is licensed under a Creative Commons Attribution 4.0 International License, which permits use, sharing, adaptation, distribution and reproduction in any medium or format, as long as you give appropriate credit to the original author(s) and the source, provide a link to the Creative Commons licence, and indicate if changes were made. The images or other third party material in this article are included in the article's Creative Commons licence, unless indicated otherwise in a credit line to the material. If material is not included in the article's Creative Commons licence and your intended use is not permitted by statutory regulation or exceeds the permitted use, you will need to obtain permission directly from the copyright holder. To view a copy of this licence, visit http://creativecommons.org/licenses/by/4.0/.

(C) The Author(s) 2021 